\title{
Structure and Energetics of Diphenylalanine Self-Assembling on $\mathrm{Cu}(110)^{\dagger}$
}

\author{
Giulia Tomba,» Magalí Lingenfelder, ${ }^{\S}$ Giovanni Costantini, ${ }^{\S, \infty}$ Klaus Kern,, , \\ Florian Klappenberger, ${ }^{\perp}$ Johannes V. Barth, ${ }^{\perp}$ Lucio Colombi Ciacchi, ${ }^{\#, \nabla}$ and \\ Alessandro De Vita*,*,\#
}

\begin{abstract}
Department of Physics, King's College London, London WC2R 2LS, United Kingdom, Max-Planck-Institut für Festkörperforschung, 70569 Stuttgart, Germany, Institut de Physique des Nanostructures, Ecole Polytechnique Fédérale de Lausanne, CH-1015 Lausanne, Switzerland, Physik Department E20, Technische Universität München, 85748 Garching, Germany, Fraunhofer Institut für Werkstoffmechanik IWM, 79108 Freiburg, Germany, Institut für Zuverlässigkeit von Bauteilen und Systemen, Universität Karlsruhe, 76131 Karlsruhe, Germany, and Centre of Excellence for Nanostructured Materials and INFM-DEMOCRITOS National Simulation Centre, 34014 Trieste, Italy
\end{abstract}

Received: August 2, 2007; In Final Form: October 18, 2007

\begin{abstract}
We investigate the dynamical features of the adsorption of diphenylalanine molecules on the $\mathrm{Cu}(110)$ surface and of their assembling into supramolecular structures by a combination of quantum and classical atomistic modeling with dynamic scanning tunneling microscopy and spectroscopic experiments. Our results reveal a self-assembling mechanism in which isolated adsorbed molecules change their conformation and adsorption mode as a consequence of their mutual interactions. In particular, the formation of zwitterions after proton transfer between initially neutral molecules is found to be the key event of the assembling process, which stabilizes the supramolecular structures. Because of the constraints on the intermolecular bonds exerted by the surface-molecule interactions, the assembly process is strictly stereoselective, and may suggest a general model for patterning and functionalization of bare metal surfaces with short chiral peptides.
\end{abstract}

\section{Introduction}

Increasing effort is currently being devoted to tailoring the structural and chemical properties of metal and semiconductor surfaces. Applications in a number of emerging technological fields, from heterogeneous catalysis to electronics and sensor development, pose novel specific requirements in terms of surface geometry and reactivity, which may be bestowed to bare surfaces via appropriate functionalization with organic molecules. The spontaneous assembly of molecular components with specifically designed functional groups is one of the most promising ways to achieve this goal. ${ }^{1}$ Among the most successful applications to date are self-assembled monolayers of alkanethiols on gold. ${ }^{2-5}$ In order to widen the range of patterns and available reactive sites, several polyfunctional building blocks are being put to trial, including peptides, which are among the most promising candidates.

Understanding the adsorption behavior of peptides onto bare or oxidized substrates is of particular relevance in the fields of biosensors and biomaterials, since the interactions of a biocompatible material with the physiological environment is normally mediated by specific peptide sequences. Although a detailed knowledge of the adsorption scenario is essential to develop

\footnotetext{
† Part of the "Giacinto Scoles Festschrift".

* Corresponding author. E-mail: alessandro.de_vita@kcl.ac.uk.

$\doteqdot$ King’s College London.

\$ Max-Planck-Institut Stuttgart.

"Ecole Polytechnique Fédérale de Lausanne.

$\perp$ Technische Universität München.

\# Fraunhofer Institut Freiburg.

$\nabla$ Universität Karlsruhe.

II Centre of Excellence for Nanostructures Materials and INFM-DEMOCRITOS Trieste.

${ }^{\infty}$ Present address: Department of Chemistry, University of Warwick, Gibbet Hill Road, Coventry, CV47AL, United Kingdom.
}

controlled and reproducible functionalization techniques, the atomic-scale features and mechanisms of dynamical supramolecular assembly processes of peptides on inorganic surfaces is still a relatively young field of research. Extensive experimental and theoretical studies have helped to shed light on the behavior of small amino acids deposited on clean surfaces under controlled conditions. ${ }^{6-10}$ More recently, similar investigations have been extended to di- and tripeptides, ${ }^{11,12}$ which represent a further step toward complex and realistic systems of biological relevance.

The aim of the present work is to provide a detailed description of the structural and energetic features that control the adsorption and assembling into supramolecular structures of diphenylalanine molecules (Phe-Phe) on the $\mathrm{Cu}(110)$ surface. These phenomena acquire a special interest in consideration of the crucial role played by the Phe-Phe structural motif in several recognition processes of biological relevance. ${ }^{13-17} \mathrm{~A}$ brief account of some results on this system has been previously reported in ref 18 . Phe-Phe is the product of the condensation reaction between two phenylalanines, which belong to the proteinogenic class of natural amino acids. Being composed of two chiral amino acid residues, diphenylalanine is itself chiral. In particular, our investigations include both the L-Phe-L-Phe and the D-Phe-D-Phe enantiomers.

Short peptides are known to interact covalently with the $\mathrm{Cu}$ (110) surface through their carboxylic and amino moieties as well as through the oxygen atom of the peptide groups. The same functional groups, though, take part in intermolecular interactions such as the formation of hydrogen bonds. Therefore, the structure and stability of amino acid supramolecular assemblies on $\mathrm{Cu}$ are determined by an equilibrium between competing surface-molecule and molecule-molecule interac- 
tions. An accurate analysis of this delicate balance requires information at the atomistic level, which can be accessed by a combination of state-of-the-art computer simulations and experimental methods. Here, we used molecular dynamics (MD) techniques at both the classical and quantum levels, focusing on the fundamental binding modes between molecule and surface and on the intermolecular interactions arising upon molecular recognition and assembling.

The simulations are used to interpret the results of highresolution dynamic scanning tunneling microscopy (STM) imaging and near-edge X-ray absorption fine structure (NEXAFS) experiments.

Our findings shed light on the relative strength of the competing interactions, explain the adsorption geometry of PhePhe on the $\mathrm{Cu}$ surface, and quantify the relative stability of different supramolecular structures. Most importantly, since the structure-determining interactions involve the nonspecific functional groups of diphenylalanine, the described scenario may also hold for other peptides, suggesting a general model for biomolecular patterning and functionalization of bare metal surfaces.

\section{Methods}

2.1. Simulation Techniques. All ab initio simulations were performed with a parallel implementation of the Car-Parrinello method ${ }^{19}$ in the LAUTREC code ${ }^{20}$ within the framework of density functional theory (DFT). We used the gradient-corrected exchange correlation potential PW9 $1^{21}$ and norm-conserving Troullier-Martins pseudopotentials ${ }^{22}$ to describe the electroncore interactions. Metallic systems were treated according to the scheme of ref 23, and the electronic states were occupied according to a Fermi-Dirac distribution with a smearing temperature of $k_{\mathrm{B}} T=0.1 \mathrm{eV}$. The scalar relativistic $\mathrm{Cu}$ pseudopotential, which takes into account 11 valence electrons, yields a lattice constant of $3.67 \AA$ and a bulk modulus of 142 GPa for crystalline copper, to be compared with experimental values of $3.61 \AA^{24}$ and $142 \mathrm{GPa}^{25}$ The simulations were performed using periodic boundary conditions, and the ksampling of the Brillouin zone was limited to the $\Gamma$-point, unless otherwise stated. The wave functions and the electron density were expanded on a plane-wave basis set up to kinetic energy cutoffs of 60 and 240 Ry, respectively. The unreconstructed $\mathrm{Cu}(110)$ surface was modeled by a periodically repeated fourlayer slab ${ }^{26}$ whose two bottom layers were kept fixed during the dynamical evolution of the system. The MD simulations were performed using a time step of $0.12 \mathrm{fs}$, and the structures were considered relaxed when all residual force components on the atoms were less than $0.03 \mathrm{eV} / \AA$. Interaction energies between adsorbed molecules and substrate (referred to for simplicity as substrate interaction energies from now on) are calculated as $E_{\mathrm{int}}=E_{\mathrm{mol} / \mathrm{Cu}(110)}-E_{\mathrm{mol}}-E_{\mathrm{Cu}(110) \text {, where }}$ $E_{\mathrm{mol} / \mathrm{Cu}(110)}$ refers to the relaxed structure of the total system, and $E_{\mathrm{mol}}$ and $E_{\mathrm{Cu}(110)}$ refer to the two isolated subsystems with no relaxation performed.

The classical MD simulations were performed with the AMBER program suite, using the generalized AMBER force field. ${ }^{27}$ The structure of Phe-Phe was initially built from the $f f 03$ database ${ }^{28}$ as a sequence of two L-phenylalanine molecules, and subsequently optimized by ab initio calculations. In the dynamical simulations, control over temperature was achieved through the weak-coupling algorithm, ${ }^{29}$ and an harmonic potential was applied to constrain the positions of selected atoms when necessary.

2.2. Simulation Models. The choice of the periodically repeated cell used in the ab initio simulations is based on the periodicity and orientation of the molecular chains that form on $\mathrm{Cu}(110)$ after exposure to Phe-Phe molecules. ${ }^{18}$ In the cell we used to model these molecular chains, two of the cell vectors are parallel to the [1 $\overline{1} 2]$ and [1 $\overline{1} \overline{2}]$ surface crystallographic directions, with dimensions of 8.89 and $22.24 \AA$, respectively. The third cell vector is perpendicular to the surface, and its length $(11.13 \AA)$ is chosen so that a vacuum layer corresponding to nine $\mathrm{Cu}$ layers separates the surface slab model from its periodically repeated images. To simulate adsorbed single molecules, the cell vector along the [1 12 ] direction is enlarged by $50 \%$ in order to avoid interactions between the molecule and its images. In the case of small test molecules, the computational cell is restricted to 2 and $3 \mathrm{Cu}-\mathrm{Cu}$ distances along the [1 $\overline{1} 2]$ and [1 $\overline{1} \overline{2}]$ directions, respectively, and the reciprocal space is sampled by four k-points.

2.3. STM Imaging. The STM experiments were performed at room temperature in an ultrahigh-vacuum chamber (base pressure, $2 \times 10^{-10}$ mbar). Clean and atomically flat surfaces of a $\mathrm{Cu}(110)$ single crystal were prepared by several cycles of ion sputtering $\left(\mathrm{Ar}^{+}\right.$ions, energy $900 \mathrm{eV}$, flux $10 \mu \mathrm{A} / \mathrm{cm}^{2}$, time $20 \mathrm{~min}$ ) followed by flash annealing to $750 \mathrm{~K}$. Phe-Phe molecules were deposited by organic molecular beam epitaxy on freshly prepared surfaces held at room temperature. L-PheL-Phe and D-Phe-D-Phe were stored in separated quartz crucibles inside Knudsen-type sources and heated to $450 \mathrm{~K}$ for sublimation. Racemic mixtures were prepared by co-deposition of both enantiomers. STM images were acquired in the constant-current mode with a typical tunneling current of $0.9 \mathrm{nA}$ and a voltage of $-2.0 \mathrm{eV}$ (filled states). The images presented here have been processed by average filtering to eliminate high frequency noise. When recording STM videos, the highest acquisition frequency was of one image every $68 \mathrm{~s}$.

2.4. NEXAFS Experiments. The NEXAFS data were taken at the HE-SGM beamline at BESSY II in Berlin. A partial electron yield mode with a retarding voltage at the carbon $\mathrm{K}$ edge of $150 \mathrm{~V}$ and at the nitrogen $\mathrm{K}$ edge of $300 \mathrm{~V}$ was used. The preparation of the $\mathrm{Cu}(110)$ substrate and the evaporation of Phe-Phe molecules were similar to those already described for the STM measurements. All spectra were recorded at room temperature and referenced against a characteristic peak in simultaneously recorded spectra of a contaminated Au grid. To extract the information related to the adsorbate layer only, the data were processed as described in ref 30 .

\section{Results}

We start our study with simulations of isolated molecules in the gas phase (section 3.1), followed by investigations of the adsorption features of single molecules (section 3.2) and of molecular chains (section 3.3). The dynamics of the assembly process and the structures of possible metastable molecular pairs are investigated in sections 3.4 and 3.5. A discussion of the results is contained in section 4 .

3.1. Gas-Phase Molecules. The charge state of peptides strongly depends on the surrounding environment. Solvated molecules can exist in neutral, ionic, or zwitterionic form, depending on the $\mathrm{pH}$ of the solution, but, upon sublimation, that is, for gas-phase molecules, neutral groups are energetically favored. To extensively sample the phase space of the molecular conformations accessible to Phe-Phe in vacuum, we followed the dynamics of a neutral isolated gas-phase molecule during a 5 ns classical MD simulation at $400 \mathrm{~K}$. The high flexibility of the molecule, which is limited only by the inherent planarity of the peptide core, (i.e., the central $\mathrm{CONH}$ group) allows it to access a wide range of conformations. These can be discrimi- 


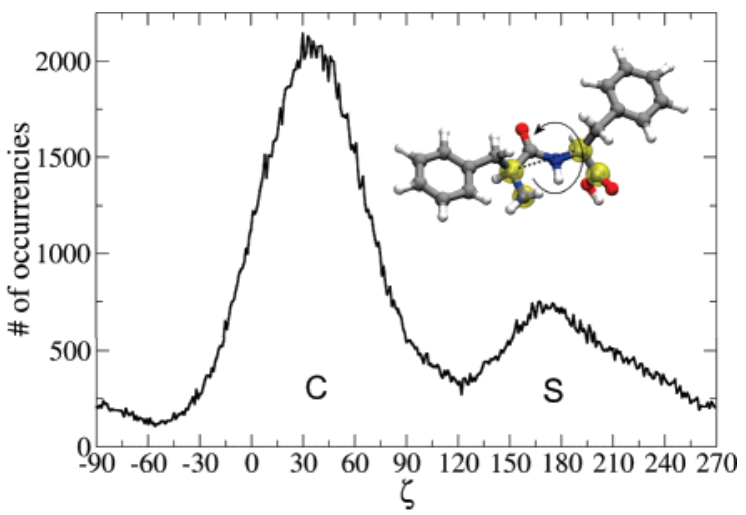

Figure 1. Distribution analysis for the torsional angle $\zeta$, defined by the four atoms highlighted in yellow in the sample C L-Phe-L-Phe conformer (upper right corner). Data refer to a classical MD simulation in gas phase at $400 \mathrm{~K}$.

nated according to the relative position of the two most important interaction sites, i.e., the $-\mathrm{COOH}$ and $-\mathrm{NH}_{2}$ groups, as quantified by the torsional angle $\zeta$ (see inset in Figure 1). The distribution of $\zeta$ during the dynamics presents two peaks centered at about $35^{\circ}$ and $170^{\circ}$ and separated by two minima at about $-50^{\circ}$ and $125^{\circ}$ (Figure 1 ). Within the first peak, both the carboxyl and amino groups are located on the same side with respect to the molecular backbone. Elsewhere, each side of the molecule hosts only one reactive functionality. We will refer to the two conformations as " $\mathrm{C}$ " and "S", respectively. As highlighted by the distribution profile in Figure 1, the molecule preferentially adopts the $\mathrm{C}$ conformation (66\% of the total simulated time).

3.2. Adsorbed Isolated Molecules. After deposition on $\mathrm{Cu}-$ (110) from the gas phase, single Phe-Phe molecules imaged with the STM (circled in Figure 2a) present a characteristic dumbbell shape in which the two terminal bumps correspond to the two phenyl rings, while the central section corresponds to the peptide backbone. The chirality of L-Phe-L-Phe or D-PheD-Phe co-deposited at room temperature at submonolayer coverages is reflected in their adsorption geometry. The main molecular axis of L-Phe-L-Phe forms an angle $\alpha_{1}=(-34 \pm$ $4)^{\circ}$ with the [1 $1 \overline{0}$ ] direction of the $\mathrm{Cu}(110)$ surface, while D-PheD-Phe adsorbs in the mirror-symmetric orientation with an angle $\alpha_{1}=(34 \pm 4)^{\circ}$ (Figure 2a, inset).

A series of ab initio MD simulations have been performed to investigate the binding mode of isolated molecules, taking into account both the $\mathrm{S}$ and the $\mathrm{C}$ conformations described in the previous section. Three different initial configurations of neutral S molecules have been considered, which lead to two different local minima after geometry relaxation (see Table 1). The first minimum corresponds to a 3-point-anchored structure in which covalent bonds with the surface are formed via both the carboxyl and the peptide $\mathrm{O}$ atoms and the amino $\mathrm{N}$ atom (Figure 3a). The calculated substrate interaction energy is 1.53 $\mathrm{eV}$, and the obtained value for the adsorption angle $\alpha_{1}$ is about $-14^{\circ}$. In the second minimum, $\alpha_{1}$ is about $-84^{\circ}$, and the interaction between the carboxylic $\mathrm{O}$ and the surface is much weaker, consistent with a lower substrate interaction energy of $1.18 \mathrm{eV}$ (Figure 3b). In both cases, the equilibrium values for $\mathrm{Cu}-\mathrm{N}$ and $\mathrm{Cu}-\mathrm{O}$ distances are in good agreement with the values reported for glycine adsorption on the same substrate. ${ }^{8}$ However, in neither case is the adsorption angle consistent with the corresponding experimental value of $-34 \pm 4^{\circ}$. Instead, a dynamical relaxation starting with a molecule in the $\mathrm{C}$ configuration lead to a minimum energy structure (Figure 4a) with an adsorption angle $\alpha_{1}$ of about $-30^{\circ}$, in much better agreement
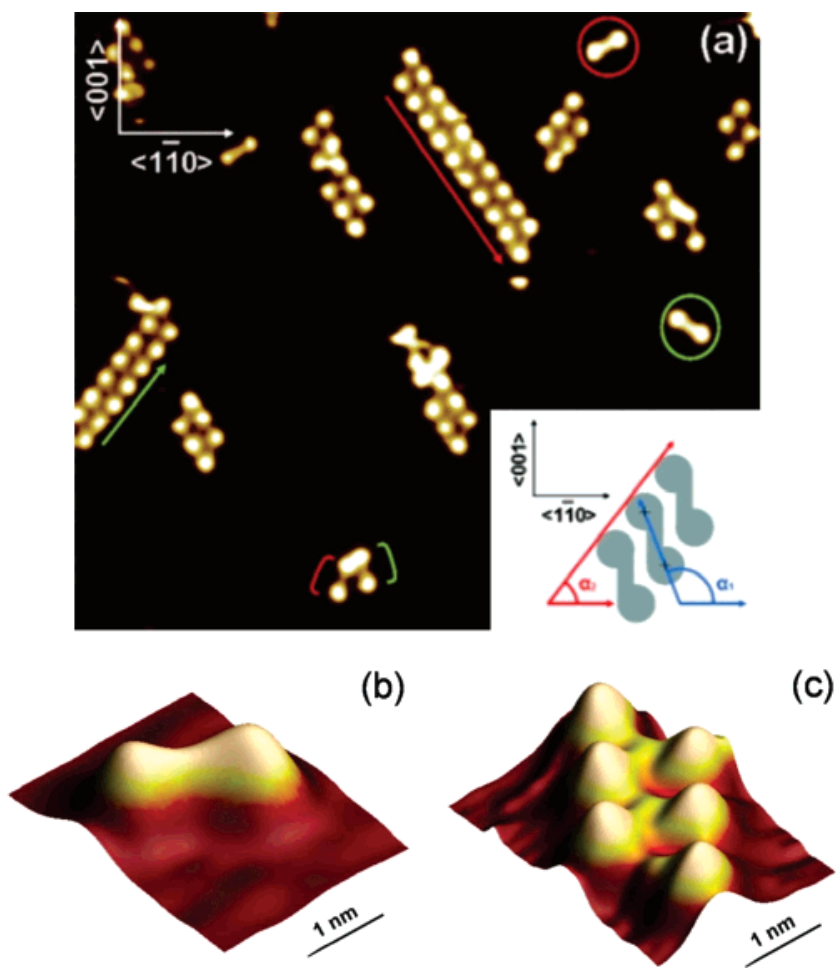

Figure 2. Racemic mixture of $\mathrm{L}-\mathrm{L}$ and $\mathrm{D}-\mathrm{D}$ diphenylalanine molecules deposited at room temperature on $\mathrm{Cu}(110)$. (a) Representative overview image. Single Phe-Phe are circled in green $(\mathrm{L}-\mathrm{L})$ and red (D-D). Homochiral supramolecular chains are indicated by arrows with the same color code. A metastable heterochiral pair is marked by open brackets at the center of the image. Inset: definition of the angles $\alpha_{1}$ (main molecular axis) and $\alpha_{2}$ (chain growth direction) with respect to the crystallographic directions. (b,c) three-dimensional rendering of $(b)$ an isolated $\mathrm{L}-\mathrm{L}$ molecule and (c) an $\mathrm{L}-\mathrm{L}$ trimer chain.

TABLE 1: Calculated Distances and Substrate Interaction Energies from the DFT-Optimized Adsorbed Systems

\begin{tabular}{|c|c|c|c|c|c|}
\hline & $\alpha_{1}$ & $\begin{array}{c}\mathrm{Cu}-\mathrm{O}_{\text {carbonyl }} \\
{[\AA]}\end{array}$ & $\begin{array}{c}\mathrm{Cu}-\mathrm{O}_{\text {carboxyl }} \\
{[\AA]}\end{array}$ & $\begin{array}{c}\mathrm{Cu}-\mathrm{N} \\
{[\AA]}\end{array}$ & $\begin{array}{c}\text { substrate } \\
\text { interaction } \\
\text { energy } \\
{[\mathrm{eV}]}\end{array}$ \\
\hline "S" Phe-Phe ${ }^{a}$ & $-84^{\circ}$ & 2.14 & 3.02 & 2.14 & 1.18 \\
\hline "S"Phe-Phe ${ }^{a}$ & $-14^{\circ}$ & 2.13 & 2.14 & 2.14 & 1.53 \\
\hline "C" Phe-Phe ${ }^{a}$ & $-30^{\circ}$ & 2.10 & 2.25 & 2.13 & 1.57 \\
\hline $\begin{array}{l}\text { "S" Phe-Phe } \\
\text { (chains) }{ }^{a}\end{array}$ & $-74^{\circ}$ & 2.35 & 2.05 & 3.32 & 1.16 \\
\hline methylamine & & & & 2.10 & 0.83 \\
\hline acetic acid & & & 2.14 & & 0.49 \\
\hline$N$-methylacetamide & & 2.09 & & & 0.56 \\
\hline
\end{tabular}

${ }^{a}$ Phe-Phe models refer to L-Phe-L-Phe enantiomers.

with the experiment. The 3-point binding mode obtained is virtually identical to what was obtained in the case of the lower energy adsorbed $\mathrm{S}$ conformation, and so is the value of the substrate interaction energy $(1.57 \mathrm{eV})$. However, the total energy of the $\mathrm{C}$ adsorbed molecule system is lower by $0.47 \mathrm{eV}$ than that in the $\mathrm{S}$ case. This is mainly due to the fact that the surface is locally strained upon the binding of Phe-Phe molecules, the amount of strain being larger for the $\mathrm{S}$ conformation than for the $\mathrm{C}$ conformation.

The individual contributions to the adsorption energy from the individual groups (i.e., their relative affinity for the $\mathrm{Cu}$ surface) can be estimated by modeling the adsorption of simple small molecules. Namely, we considered acetic acid, methylamine, and $\mathrm{N}$-methylacetamide, which contain the $-\mathrm{COOH}$, $-\mathrm{NH}_{2}$, and $\mathrm{CONH}$ groups, respectively. The initial positions of the molecules on the substrate were chosen according to the 

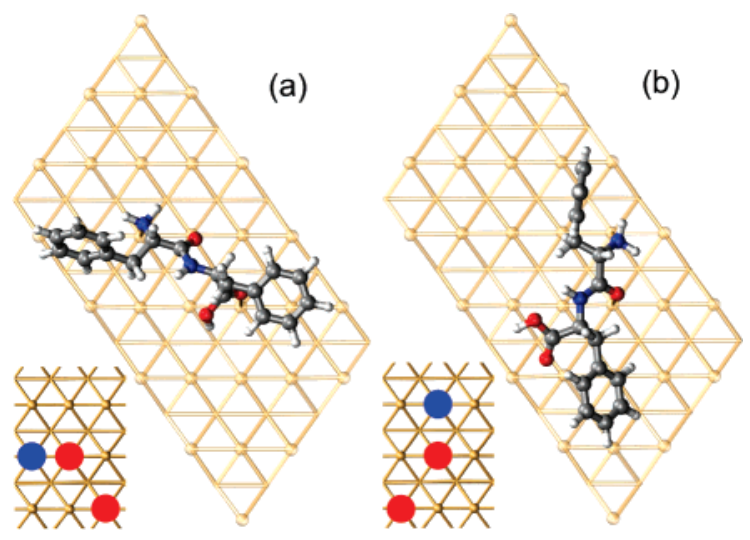

Figure 3. DFT models for the adsorption of a single L-Phe-L-Phe molecule, S conformation. Two different local minima are obtained, characterized by an angle $\alpha_{1}$ of about $-14^{\circ}$ (a) and $-84^{\circ}$ (b). Insets: 3-point simplified binding schemes; red and blue circles respectively identify the $\mathrm{Cu}$ atoms to which oxygen and nitrogen atoms of the molecule bind upon adsorption. While orientation "a" allows the two involved oxygens and the nitrogen to lie in "atop" geometries, in orientation "b", the more weakly bound oxygen is shifted toward a near "bridge" position.
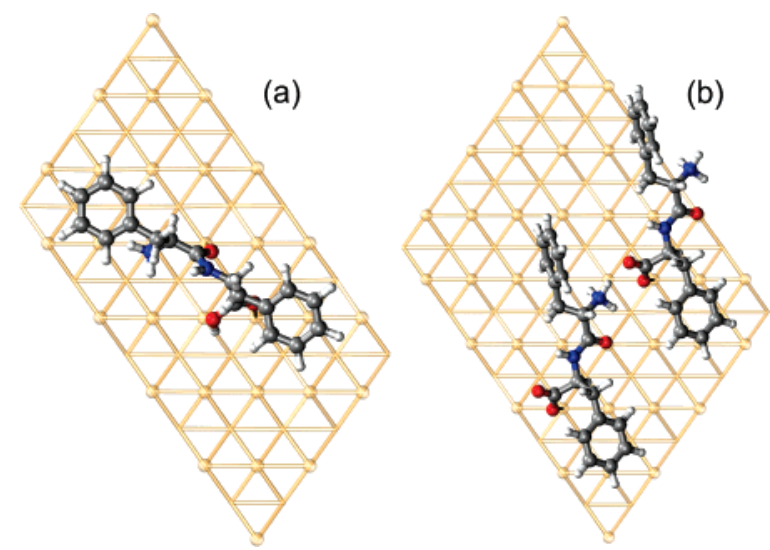

Figure 4. DFT-optimized models for the adsorption of (a) a single L-Phe-L-Phe molecule, C conformation, and (b) L-Phe-L-Phe molecules, $\mathrm{S}$ conformation, as part of a supramolecular chain (two repeated unit cells are represented).

arrangement of the respective groups in the $C$-diphenylalanine system, after which their adsorption geometries were separately optimized. The geometric features and substrate interaction energies for the final relaxed structures are summarized in Table 1 , revealing that the amino group interacts most strongly with the surface, followed by the carbonyl and the carboxyl groups. The chemical interaction effects of the adsorption are highlighted by the analysis of the spatially resolved electron density difference, defined as $\Delta \rho(r)=\rho_{\text {mol } / \text { slab }}(r)-\left[\rho_{\text {mol }}(r)+\rho_{\text {slab }}(r)\right]$, where $\rho_{\mathrm{mol} / \mathrm{slab}}$ represents the electronic density of the complete system, and $\rho_{\text {mol }}$ and $\rho_{\text {slab }}$ are the densities of the noninteracting molecule and surface constituents (Figure 5) in the same geometries as in the interacting system.

3.3. Adsorbed Molecular Chains. Upon deposition of PhePhe on $\mathrm{Cu}(110)$ at room temperature, the isolated molecules assemble into supramolecular structures in the form of chains (indicated with arrows in Figure 2a). Interestingly, these chains only contain molecules bearing the same chirality, and can be discriminated on the basis of their geometrical properties. ${ }^{18}$ Namely, the molecules are adsorbed with an angle $\alpha_{1}$ of -74 $\pm 4^{\circ}$ within $\mathrm{L}-\mathrm{L}$ chains, and of $74 \pm 4^{\circ}$ within $\mathrm{D}-\mathrm{D}$ chains (Figure 2a). Moreover, the angle $\alpha_{2}$ formed between the chain (a)

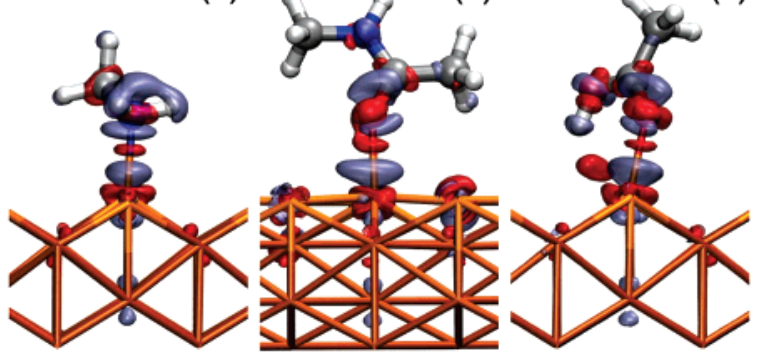

Figure 5. Electronic density difference plots for (a) methylamine, (b) $\mathrm{N}$-methylacetamide, and (c) acetic acid adsorbed on $\mathrm{Cu}(110)$. The isosurfaces correspond to a value of $\pm 0.02 \mathrm{C} / \mathrm{cm}^{3}$ (red: charge enrichment; blue: charge depletion).

axis and the [1실 surface direction is equal to $52 \pm 2^{\circ}$ and $-52 \pm 2^{\circ}$ for $\mathrm{L}-\mathrm{L}$ and $\mathrm{D}-\mathrm{D}$ molecules, respectively.

The formation of hydrogen bonds between the carboxyl and the amino groups of different Phe-Phe molecules may lead to several kinds of supramolecular assemblies depending on the relative molecular conformations. ${ }^{18}$ In particular, two molecules with $\mathrm{C}$ conformation would saturate their functionalities by forming a doubly bound molecular pair, while molecules with $\mathrm{S}$ conformation could assemble in linear structures of arbitrary length. Indeed, in a classical gas-phase MD simulation, we observed a series of nine L-Phe-L-Phe molecules bound to each other through the carboxylic and amino moieties, forming a stable, open-ended chain. We thus performed an ab initio gasphase MD simulation placing a single $S$ molecule in a periodically repeated cell with the translational symmetry of the $\mathrm{Cu}(110)$ lattice. A stable hydrogen bond between the carboxyl group of the molecule and the amino group of its adjacent periodic image forms during a 590 fs damped dynamics simulation. This bond ensures the stability of the "infinite" periodic chain of neutral molecules. The obtained $d_{\mathrm{N}-\mathrm{O}}$ hydrogen bond distance is $2.73 \AA$, and the calculated bond energy is 0.43 $\mathrm{eV}$. The predicted angle between the gas-phase chain growth direction and the main molecular axis is $-112^{\circ}$. The corresponding $\left(\alpha_{1}-\alpha_{2}\right)$ angle observed in the STM experiments on the $\mathrm{Cu}$ surface is $-126^{\circ}$. The discrepancy of $14^{\circ}$ may be due to the additional constraints exerted on the Phe-Phe molecule by its specific adsorption sites, influencing the supramolecular structure.

To investigate this issue, we performed a new ab initio simulation where the relaxed gas-phase chain structure was positioned on the surface. Consistent with the adsorption mode of single molecules described above, we initially assigned to the oxygen atoms of the carboxyl and carbonyl groups nearly "atop" positions with respect to surface $\mathrm{Cu}$ atoms, which resulted in an initial adsorption angle $\alpha_{1}=-58^{\circ}$. The trajectory of the system was analyzed during an unconstrained MD simulation followed by slow damping of the atomic motion, eventually leading to the equilibrium structure of Figure $4 \mathrm{~b}$ after a total simulated time of about $4.2 \mathrm{ps}$. From the initial to the final configuration, the molecule undergoes a substantial rearrangement characterized by a shift of the adsorption angle $\alpha_{1}$ to $-74^{\circ}$, in agreement with the value of $-74 \pm 4^{\circ}$ measured with STM. In the final relaxed structure, the $\mathrm{O}$ atoms are still very close to "atop" sites, with equilibrium $\mathrm{Cu}-\mathrm{O}$ distances of 2.05 and 2.35 $\AA$ for the carboxyl and carbonyl $\mathrm{O}$ atoms, respectively. Interestingly, during the dynamics, the peptide carbonyl oxygen is observed to slide from one surface adsorption site to the next along the [1 $1 \overline{1} 0]$ crystallographic direction, suggesting a smooth potential energy surface for diffusion along this direction. At 


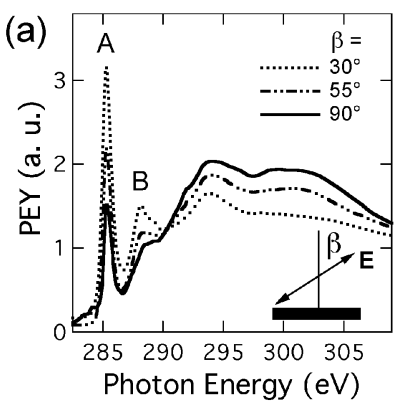

(c)

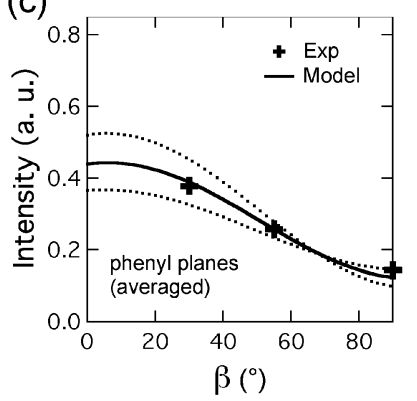

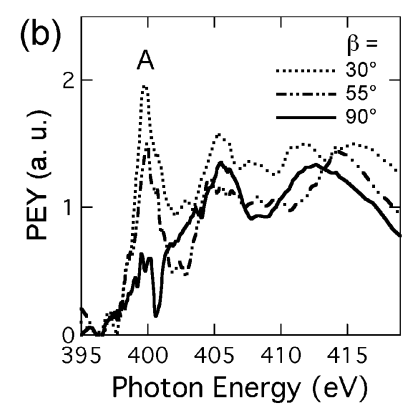

(d)

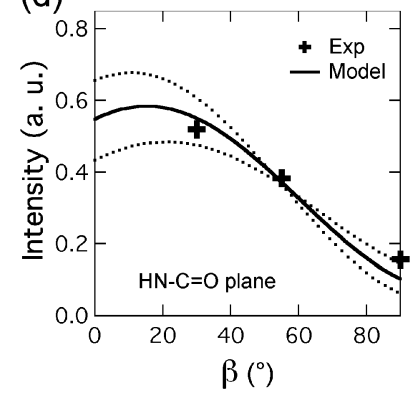

Figure 6. NEXAFS spectra of the $\mathrm{C} 1 \mathrm{~s}$ edge (a) and of the N1s edge (b) of chains of D-Phe-D-Phe and comparison between the theoretical prediction (solid line) and the experimental intensities (crosses) for the average of the phenyl planes (c) and the peptide plane (d). Theoretical predicted curves for geometries deviating by $\pm 10^{\circ}$ from the DFToptimized structure are reported for comparison (dotted lines). The nitrogen data suffer from noise due to an unstable detector background.

the same time, the amino group approaches the substrate up to a final $3.32 \AA \mathrm{Cu}-\mathrm{N}$ distance, while maintaining the hydrogen bond with the nearby carboxylic group throughout the trajectory.

Furthermore, we observed spontaneous proton transfer from the carboxylic group of the molecule to the amino moiety of its neighbor along the chain, yielding a chain of zwitterions. Forcing the $\mathrm{H}$ atom back to the carboxyl group resulted in a $0.45 \mathrm{eV}$ increase of the total energy of the system, suggesting that the stable chemical state of Phe-Phe molecules forming supramolecular chains is indeed zwitterionic. In the final chain structure, the substrate interaction energy per molecule is 1.16 $\mathrm{eV}$, that is, $0.41 \mathrm{eV}$ less than that in the stably adsorbed isolated $\mathrm{C}$ molecules (see Table 1). Since the calculated energy gain associated with the formation of a chain of neutral molecules is only $0.43 \mathrm{eV}$ (see above), the observed proton-transfer event may be essential to provide a net thermodynamic driving force for the formation of stable molecular chains. This issue will be further discussed in section 4.

The chemical state and the structural details of the supramolecular chains were investigated via X-ray photoelectron spectroscopy (XPS) and NEXAFS spectroscopy after deposition of Phe-Phe at a coverage of about $1 \mathrm{ML}$, at which most molecules have assembled into chain structures. ${ }^{31}$ As already reported in ref 18 , the XPS data are consistent with a zwitterionic charge state of the assembled Phe-Phe molecules. The NEXAFS spectra of the $\mathrm{C} 1 \mathrm{~s}$ edge (Figure $6 \mathrm{a}$ ) show two broad $\sigma^{*}$ features and two $\pi^{*}$ resonances. The first one (peak A in Figure 6a) is attributed to the lowest lying phenyl resonance, ${ }^{30,32,33}$ while the second one (peak B in Figure 6a) consists of a mixture of higher lying phenyl resonances and will therefore not be taken into account in the following analysis. The N1s edge features a single $\pi^{*}$ resonance (labeled $\mathrm{A}$ in Figure $6 \mathrm{~b}$ ) stemming from the peptide subunit $(\mathrm{CONH}) .{ }^{33}$ The spectra for both $\mathrm{C}$ and $\mathrm{N}$ show a distinct dependence on the radiation incidence angle $\beta$ (see Figure 6 , inset), which, in our experiments, was set to $30^{\circ}, 55^{\circ}$, and $90^{\circ}$.

The intensity variations measured by NEXAFS are directly related to the geometrical features of the system, since each molecular resonance scales with the angle between the corresponding transition dipole moment and the radiation incidence direction. Starting from a known structure, the relationship between these values can also be used to derive a corresponding expected trend for $I(\beta) .{ }^{34} \mathrm{We}$ applied a similar procedure to our DFT-derived adsorption geometry in order to check the consistency of our model with the experimental data. The averaged intensity curves corresponding to the two phenyl rings are reported in Figure $6 c$, while the intensity curve for the peptide group is shown in Figure 6d (solid lines). In order to investigate the angular sensitivity of the method, two further theoretical curves (dotted lines) were calculated for adsorption angles which differ by $\pm 10^{\circ}$ with respect to the equilibrium geometry. The normalized experimental intensities (crosses) are in good agreement with the data derived from the equilibrium structure, clearly providing the best fit among the three curves.

3.4. The Process of Supramolecular Assembling. We followed the formation, growth, and dissociation dynamics of several Phe-Phe chains by acquiring consecutive STM images on the same surface region (videos V1-V3 in Supporting Information). The mutually induced, stereoselective formation of molecular dimers has been reported and discussed in ref 18 . Starting from a pre-existing dimer (red circle in Figure 7(i)a), the interaction with a third molecule (indicated by a red arrow in Figure 7(i)) takes place initially with the formation of a metastable trimer structure (Figure $7(\mathrm{i}) \mathrm{d}-\mathrm{h}$ ). Later, stabilization of the interaction between the new molecule and its neighbor causes the temporary disruption of the initial pair (Figure 7(i)ij), eventually followed by the formation of a stable trimer (Figure 7(i)k-1). A similar disruption event was also observed during the formation of a tetramer (Figure 7(ii)a-h). In this case, a fourth molecule approaches a pre-existing trimer and binds to one of its end molecules. This initially leads to separation into two dimers, which only later join again to form a tetramer. Interestingly, the tendency of a newly approaching molecule to induce the breaking-up of the last bond in a chain is less frequently observed with increasing chain length. Namely, we never observed such an event for chains composed of nine or more molecules (Figure 7(iii)a-d), although we could sometimes observe a longer chain spontaneously lose its last molecule. However, the spontaneous dissociation of homochiral dimers into separated molecules was never recorded in our experiments, pointing toward a very high stability of molecular pairs.

3.5. Metastable Molecular Pairs. As mentioned above, the process of molecular assembling is strictly stereoselective. Occasionally, heterochiral pairs can also be found in the STM images (cf., e.g., the lower part of Figure 2a), but their lifetime is much shorter than that for homochiral pairs. In addition, metastable homochiral pairs are also observed, mainly during the process of mutual recognition and assembling of two individual molecules. Examples of such metastable pair structures are reported in the insets of Figure 8. Their adsorption geometries are indicative of different intermolecular interactions, which reflect the presence of several functionalities in PhePhe.

$\mathrm{Ab}$ initio simulations of such adsorbed structures would be computationally too demanding, but valuable insight into the binding modes can be provided by gas-phase classical MD simulations incorporating the information obtained with the DFT simulations described in the previous sections. Namely, relaxed $\mathrm{ab}$ initio configurations of adsorbed molecules were used as 
i)

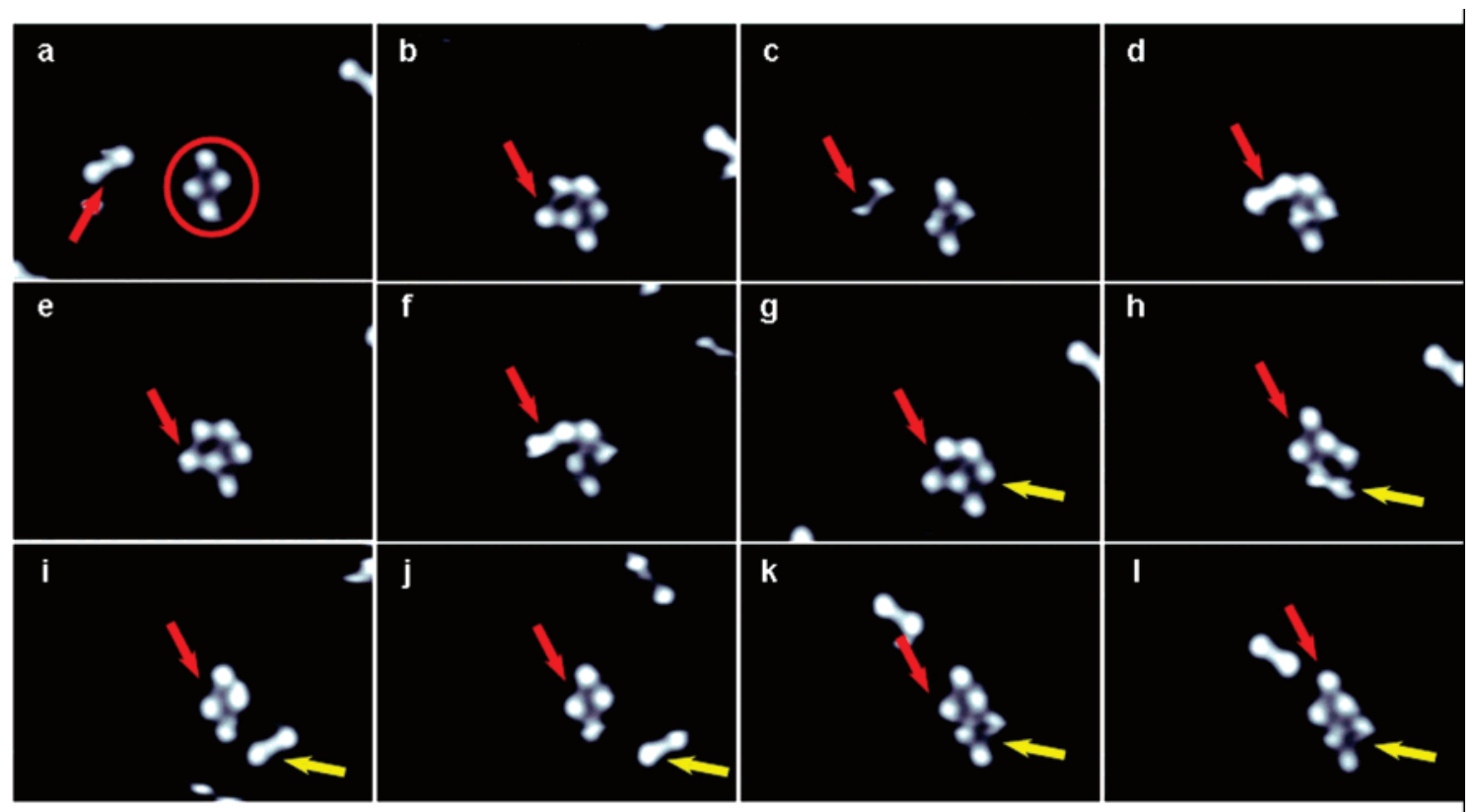

ii)
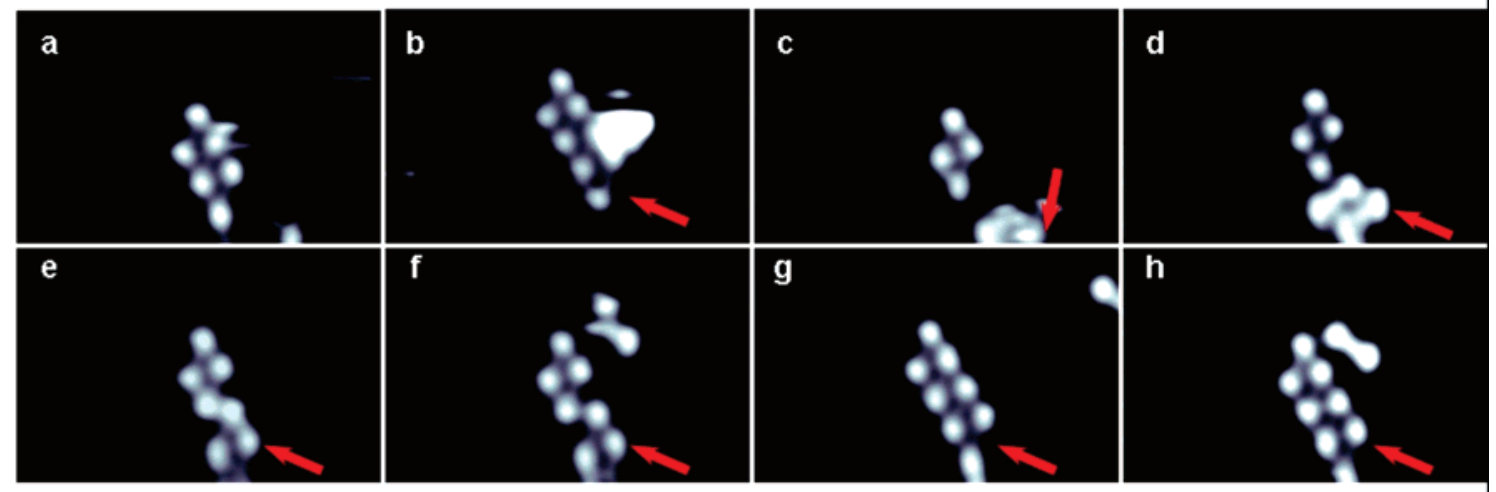

iii)
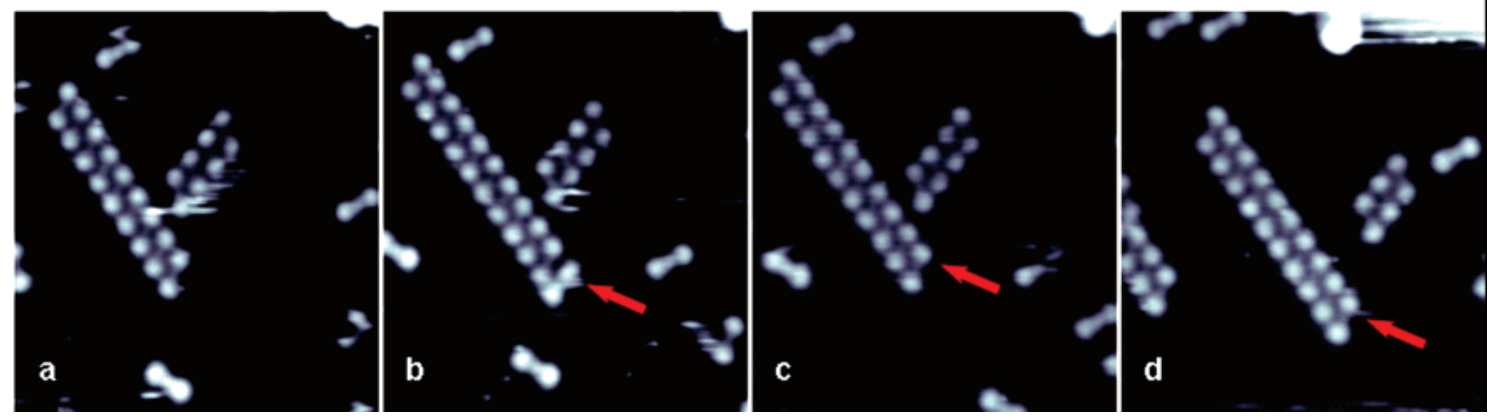

Figure 7. Selected frames of three STM videos showing the formation path of homochiral chains composed of three, four, and ten D-Phe-D-Phe molecules in groups (i), (ii), and (iii), respectively. Timing (in seconds): (i) $a=0, b=68, c=136, d=204, e=340, f=408, g=680, h=$ $748, i=816, j=952, k=1292, l=1350$; (ii) $a=0, b=68, c=136, d=408, e=476, f=1020, g=1360, h=1496$; (iii) $a=0, b=68$, $d=136, e=3060$. Joining molecules are indicated by red arrows, while the yellow arrows point to a dimer molecule that temporarily detaches from the growing structure.

initial structures, and harmonic restraints were applied on the oxygen or nitrogen atoms in order to mimic the effect of the covalent bond to the surface adsorption sites. The initial relative distance and mutual orientation of the molecules were set to be commensurate with the $\mathrm{Cu}(110)$ substrate at the calculated stable adsorption angles. Since the presence of zwitterions is presumably associated with stable dimers or chains, only neutral molecules were considered here to simulate metastable pairs.

Classical MD simulations of both homochiral and heterochiral pairs were performed at temperatures up to $200 \mathrm{~K}$, each for about 200 ps, after which the structures were relaxed to their 0 $\mathrm{K}$ equilibrium geometry. The so-obtained molecular pair structures contain hydrogen bonds either between the carboxyl and peptide carbonyl groups (see Figure 8) or between the peptide carbonyl and amino groups. Moreover, we repeatedly observe interactions between the amino or carboxylic groups and the phenyl rings, especially in the case of homochiral pairs. An ab initio simulation of a benzene and a glycine molecule in gas phase allows us to estimate the energy of these interactions: we find that the system is stabilized by 0.14 or $0.18 \mathrm{eV}$ when the delocalized $\pi$-electrons of benzene interact with the amino or the carboxylic side of glycine, respectively (see Figure $9)$. We note that cation $-\pi$ interactions are known to promote inter- and intramolecular stabilization in biological macromolecules. ${ }^{35,36}$ 

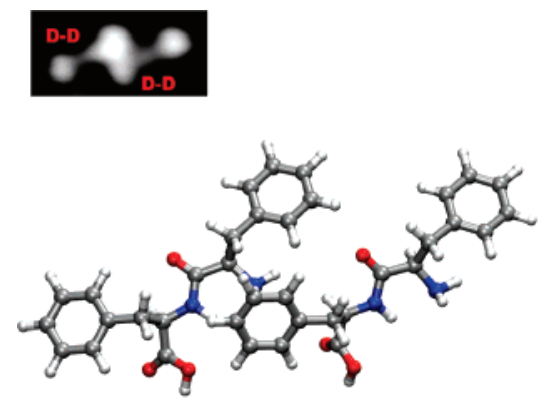

(a)
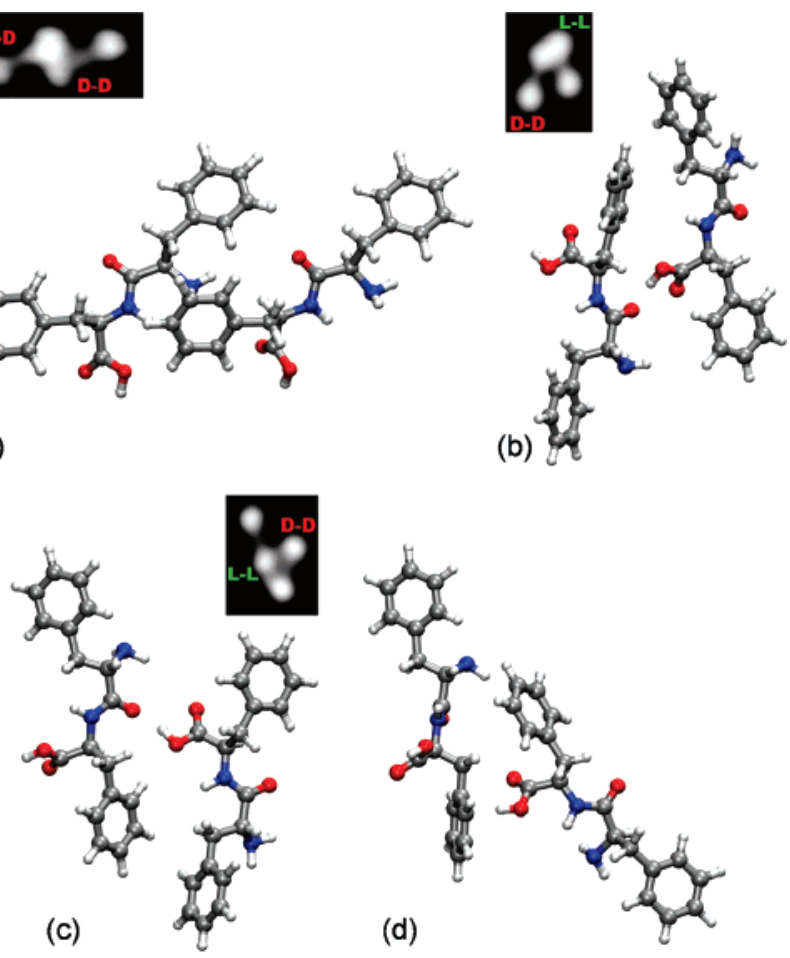

Figure 8. Pair structures obtained from classical MD simulations. Comparable pairs observed in STM images are represented as insets. A range of molecule - molecule interactions are observed, from $-\mathrm{NH}_{2} /$ phenyl or $-\mathrm{COOH} /$ phenyl interactions (homochiral pair (a), heterochiral pair (d)) to hydrogen bonds between the carboxylic and carbonyl groups $(\mathrm{b}, \mathrm{c})$.
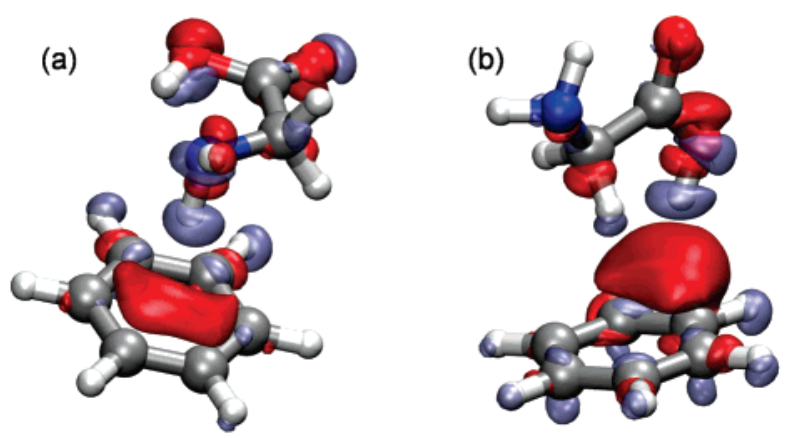

Figure 9. Electronic density difference plots showing the effect of the interaction between a benzene molecule and the amino (a) or the carboxylic (b) group of a glycine molecule. The depicted isosurfaces correspond to a value of $\pm 0.0045 \mathrm{C} / \mathrm{cm}^{3}$ (red: charge enrichment; blue: charge depletion).

\section{Discussion}

On the basis of the presented results, we can conclude that isolated neutral molecules adsorb from the gas phase on the $\mathrm{Cu}$ surface in the $\mathrm{C}$ conformation with the amino and carboxyl functional groups located on the same side with respect to the molecular axis. As shown by STM imaging, diffusion processes occur at room temperature, and lead to mutual interactions between single molecules. This results in their initial assembly into pairs and in the subsequent growth of longer chains by the addition of single molecules to existing chains (see Figure 7). A conformation change in which the two functional groups move to opposite sides of the molecular axis (S conformation), accompanied by a rotation of the molecular axis with respect to the surface, takes place during the formation of molecular pairs and chain growth. In this process, the $\mathrm{CONH}$ peptide core of the molecule moves from an orientation nearly perpendicular to one nearly parallel to the $\mathrm{Cu}$ surface. This is visible in the relaxed DFT models of adsorbed molecules (Figures $4 \mathrm{a}$ and $4 \mathrm{~b}$ ) and in the STM images, which show a higher STM signal intensity in the central backbone region of $\mathrm{C}$ molecules relative to that in S molecules (cf. Figure 2, panels b and c).

An analysis of the individual contributions to the single molecule adsorption energy quantified using small model systems reveals that the strongest molecule-surface interaction occurs through the amino group (Table 1), whereas the carboxylic group is the most weakly bound. ${ }^{37}$ Furthermore, when single molecules assemble into chains, the hydrogen bond formation between the amino group of one molecule and the carboxyl group of the next molecule is accompanied by proton transfer, leading to a chain of zwitterions. In the chain geometry, the molecular amino groups are no more covalently bound with the metal substrate, so that each molecule in the chain is significantly less bound to the surface than an isolated molecule. At the same time, a comparable energy gain is associated with the formation of the hydrogen bond between molecules inside the chain. If we assume that the chained molecules still bear neutral functional groups, the calculated values of these two energy contributions are 0.41 and $0.43 \mathrm{eV}$ per molecule, respectively, virtually indistinguishable within an expected DFT error of about $0.1 \mathrm{eV}$. However, our results suggest that proton transfer can stabilize the chain structure by a further calculated $0.45 \mathrm{eV}$ per molecule, thus providing the necessary driving force to form chains. We note that a similar zwitterionic bonding scheme has been recently reported for methionine assembling on $\operatorname{Ag}(111){ }^{38}$

From the energy results above, a lower limit to the energy barrier for the dissociation of a molecule from a chain can be estimated to be $0.47 \mathrm{eV}$. However, as described above, dissociation events are observed when a new molecule approaches a pre-existing dimer or trimer and interacts with one of their end molecules. The two molecules at the ends of each zwitterionic chain are expected to be in different chemical states than those located inside the chain. Namely, they can be in either an acidic, positively charged state (containing a $\mathrm{NH}_{3}{ }^{+}$and a $\mathrm{COOH}$ group) or in a basic, negatively charged state (containing a $\mathrm{NH}_{2}$ and $\mathrm{COO}^{-}$group). When a terminal molecule of a chain binds to a newly added molecule, the proton-transfer process changes its state from charged to zwitterionic. Its back-bonding to the chain is expected to get weakened in this process, so that a temporary spontaneous breaking off of a dimer at a growing end of a chain is expected to occur, at least for short chain segments where the relatively low number of aligned molecular dipoles is not sufficient to electrostatically stabilize any further joining molecule directly upon addition. ${ }^{39}$

During the assembling process, a reorientation of the molecules from a $\mathrm{C}$ to an $\mathrm{S}$ conformation occurs as a result of mutually induced molecule-molecule interactions. ${ }^{18}$ For instance, during the formation of a homochiral dimer, a set of STM images taken in rapid succession on the same sample at room temperature shows that the two interacting molecules switch back and forth from the $\mathrm{C}$ to the $\mathrm{S}$ conformation (changing their adsorption angle from $34^{\circ}$ to $74^{\circ}$ and back) three times in about $8 \mathrm{~min}$ before reaching their final stable dimer configuration. Although very limited in time, this experiment provides us with a rough estimate of the rate $k_{\mathrm{f}}$ of the conformational change necessary for the assembly. This rate is related to the energy barrier $E$ associated with the conformational change by the Arrhenius equation $k_{\mathrm{f}}=k_{0} \exp \left(-E / k_{\mathrm{B}} T\right)$, where $k_{\mathrm{B}}$ is the Boltzmann constant. Assuming a value of $10^{11}-10^{12}$ $\mathrm{s}^{-1}$ for the prefactor $k_{0}$ (consistent with the attempt frequency 
associated with a relatively soft phonon mode ${ }^{40}$ ), the energy barrier $E$ can be estimated to be about $0.8 \mathrm{eV}$. This value is possibly overestimated because of undersampling, as some more switching events may have been missed as a result of our limited image acquisition rate.

The energy barrier associated with the $\mathrm{C}$ to $\mathrm{S}$ transition of two Phe-Phe molecules interacting but not yet irreversibly bound to each other can be also roughly estimated as twice the substrate interaction energy difference between isolated $\mathrm{C}$ and $\mathrm{S}$ molecules adsorbed in orientations similar to the ones observed during the assembling process. This gives a value for $E$ of 0.78 $\mathrm{eV}$. This is again only an indicative value, as a small kinetic barrier could accompany the reverse $\mathrm{S}$ to $\mathrm{C}$ transition, while the concerted transition energy cost will be reduced by the interaction between the two molecules (as indeed expected since these transitions are only observed during such an interaction).

While these two estimates are consistent and broadly comparable, we note that the theoretical estimate mostly originates from the weakening of the molecule-surface interaction during the $\mathrm{C}$ to $\mathrm{S}$ transition. Indeed, it appears likely that breaking the bonds with the substrate could be the bottleneck event associated with the switching from a $\mathrm{C}$ to an $\mathrm{S}$ conformation and the change of the adsorption angle from $34^{\circ}$ to $74^{\circ}$. Other energy barriers could be involved in the conformation switch. These include the barriers associated with the torsional angle change in the molecular backbone and those encountered during the sliding of the peptide oxygen along the compact row of [110] $\mathrm{Cu}$ atoms to allow the adsorption angle $\alpha_{1}$ change. However, both sets of barriers are expected to be low, as transitions between $\mathrm{C}$ and $\mathrm{S}$ conformations are readily observed in our gas-phase simulations, while the peptide group sliding also occurs spontaneously in our DFT simulations on adsorbed molecules well below room temperature.

\section{Conclusions}

Our results suggest that stably adsorbed $\mathrm{C}$ Phe-Phe conformers undergo a change of conformation and adsorption bonding geometry if and only if they meet and interact. Thus, our results provide evidence that the free energy landscape associated with each molecule is significantly modified by the interaction, and, in particular, the energy barrier associated with the conformation change is expected to decrease significantly if a Phe-Phe adsorbed molecule meets and binds with another similar molecule of the same chirality. In this case, the interaction eventually stabilizes both molecules in the $S$ conformation, which provides a better mutual binding geometry. We note that a similar "induced fit" mechanism has been proposed long ago in the context of biological molecules. ${ }^{41,42}$ In our case, the monomers ability to switch conformation acquires a functional character in that it drives the self-assembly of ordered enantiopure supramolecular structures. The key event leading to the stabilization of molecular dimers and chains is the transfer of a proton between the carboxyl and amino groups of adjacent assembling molecules. This leads to the formation of zwitterionic molecular chains, whose charge state has been confirmed in XPS experiments and whose predicted molecular structure is consistent with NEXAFS data.

Acknowledgment. We thank Thomas Strunskus and Christof Wöll for their assistance during the NEXAFS experiments. A.D.V., J.V.B., and K.K. acknowledge support from the ESFEUROCORES SONS Programme. CPU time allocation has been provided by the HPCx UK supercomputing facilities, by the Rechenzentrum Garching (Germany) through the German
Max-Planck Society, and by the Hochleistungsrechenzentrum Stuttgart (Germany) through the HPC-EUROPA project (RII3CT-2003-506079, with the support of the European Community - Research Infrastructure Action of the FP6). G.T. acknowledges financial support from the Garlick Scholarship Foundation. The work of L.C.C. has been supported by the Alexander von Humboldt Stiftung and by the Deutschen Forschungsgemeinschaft within the Emmy Noether Programme.

Supporting Information Available: Room-temperature STM videos focusing on the adding up of one molecule to homochiral superstructures of different lengths: a dimer (V1), a trimer (V2), and a 9-mer chain (V3). This material is available free of charge via the Internet at http://pubs.acs.org.

\section{References and Notes}

(1) Barth, J. V. Annu. Rev. Phys. Chem. 2007, 58, 375

(2) Camillone, N.; Chidsey, C. E. D.; Liu, G.; Scoles, G. J. Chem. Phys. 1993, 98, 3503

(3) Lavrich, D. J.; Wetterer, S. M.; Bernasek, S. L.; Scoles, G. J. Phys. Chem. B 1998, 102, 3456.

(4) Vargas, M. C.; Giannozzi, P.; Selloni, A.; Scoles, G. J. Phys. Chem. B 2001, 105, 9509 .

(5) Liang, J.; Scoles, G. Langmuir 2007, 23, 6142.

(6) Barlow, S. M.; Kitching, K. J.; Haq, S.; Richardson, N. V. Surf. Sci. 1998, 401, 322.

(7) Nyberg, M.; Hasselström, J.; Karis, O.; Wassdahl, N.; Weinelt, M.; Nilsson, A. J. Chem. Phys. 2000, 112, 5420

(8) Hasselström, J.; Karis, O.; Weinelt, M.; Wassdahl, N.; Nilsson, A.; Nyberg, M.; Pettersson, L. G. M.; Samant, M. G.; Stör, J. Surf. Sci. 1998, 407, 221.

(9) Rankin, R. B.; Sholl, D. S. Surf. Sci. 2005, 574, L1.

(10) Kühnle, A.; Linderoth, T. R.; Hammer, B.; Besenbacher, F. Nature 2002, 415, 891 .

(11) Barlow, S. M.; Raval, R. Surf. Sci. Rep. 2003, 50, 201

(12) Stensgaard, I. Surf. Sci. 2003, 545, L747.

(13) Kirchhausen, T. Nat. Rev. Mol. Cell Biol. 2000, 1, 187.

(14) Fiedler, K.; Veit, M.; Stamnes, A.; Rothman, J. E. Science 1996 $273,1396$.

(15) Sunde, M.; Blake, C. C. Q. Rev. Biophys. 1998, 31, 1

(16) Dobson, C. M. Trends Biochem. Sci. 1999, 24, 329.

(17) Gazit, E. Angew. Chem., Int. Ed. 2002, 41, 257.

(18) Lingenfelder, M.; Tomba, G.; Costantini, G.; Colombi Ciacchi, L.; De Vita, A.; Kern, K. Angew. Chem., Int. Ed. 2007, 46, 4492.

(19) Car, R.; Parrinello, M. Phys. Rev. Lett. 1985, 55, 2471.

(20) Vladimirova, M.; Stengel, M.; De Vita, A.; Baldereschi, A.; Boeringer, M.; Morgenstern, K.; Berndt, R.; Schneider, W.-D. Europhys. Lett. 2001, 56, 254.

(21) Perdew, J. P.; Wang, Y. Phys. Rev. B 1992, 45, 13244.

(22) Troullier, N.; Martins, J. L. Phys. Rev. B 1991, 43, 1993

(23) Stengel, M.; De Vita, A. Phys. Rev. B 2000, 62, 15283.

(24) American Institute of Physics Handbook, 3rd ed.; McGraw-Hill: New York, 1972.

(25) van't Klooster, P.; Trappeniers, N. J.; Biswas, S. N. Physica B 1979, 97,65 .

(26) We verified that no significant change takes place upon increasing the number of layers from 4 to 6 (the surface energy differs by $\sim 0.01 \mathrm{eV}$ ). The value for the surface energy of the unit cell, sampled with a $6 \times 8 \times$ $1 \mathrm{k}$-grid reduced according to Monkhorst-Pack, is $1.43 \mathrm{~J} / \mathrm{m}^{2}$, whereas the experimental value is $1.77 \mathrm{~J} / \mathrm{m}^{2}$ (as reported in Kallinteris, G. C.; Papanicolaou, N. I.; Evangelakis, G. A. Phys. Rev. B 1997, 55, 2150).

(27) Wang, J.; Wolf, R. M.; Caldwell, J. W.; Kollman, P. A.; Case, D. A. J. Comput. Chem. 2004, 25, 1157.

(28) Duan, Y.; Wu, C.; Chowdury, S.; Lee, M. C.; Xiong, G.; Zhang, W.; Yang, R.; Cieplak, P.; Luo, R.; Lee, T. J. Comput. Chem. 2003, 24, 1999.

(29) Berendsen, H. J. C.; Postma, J. P. M.; van Gunsteren, W. F.; DiNola, A.; Haak, J. R. J. Chem. Phys. 1984, 81, 3684.

(30) Cañas-Ventura, M. E.; Klappenberger, F.; Clair, S.; Pons, S.; Kern, K.; Brune, H.; Strunskus, T.; Wöll, C.; Fasel, R.; Barth, J. V. J. Chem. Phys. 2006, 125, 184710 .

(31) Lingenfelder, M. (Max-Planck-Institut für Festkörperforschung, Stuttgart, Germany) Klappenberger, F. (Technische Universität München, Garching, Germany); Classen, T. (Max-Planck-Institut für Festkörperforschung, Stuttgart, Germany); Strunskus, T. (Lehrstuhl für Physikalische Chemie I, Ruhr-Universität Bochum, Bochum, Germany); Wöll, C. (Lehrstuhl für Physikalische Chemie I, Ruhr-Universität Bochum, Bochum, Germany); Tomba, G. (King's College London, London, U.K.); De Vita, 
A. (King's College London, London, U.K.); Barth, J. V. (Technische Universität München, Garching, Germany); Costantini, G. (Max-PlanckInstitut für Festkörperforschung, Stuttgart, Germany); Kern, K. (Max-PlanckInstitut für Festkörperforschung, Stuttgart, Germany) . To be submitted for publication.

(32) Käfer, D.; Ruppel, L.; Witte, G.; Wöll, C. Phys. Rev. Lett. 2005, 95,166602

(33) Klappenberger, F.; Cañas-Ventura, M. E.; Clair, S.; Pons, S.; Schlickum, U.; Qu, Z.-R.; Brune, H.; Kern, K.; Strunskus, T.; Wöll, C. Comisso, A.; De Vita, A.; Ruben, M.; Barth, J. V. ChemPhysChem 2007, 8, 1782

(34) Stöhr, J. NEXAFS Spectroscopy; Springer: Heidelberg, Germany, 1991.

(35) Dougherty, D. A. Science 1996, 271, 163.

(36) Steiner, T. Angew. Chem., Int. Ed. 2002, 41, 48.
(37) This is further supported by a DFT simulation of the adsorbed C molecule at $350 \mathrm{~K}$, where the carboxyl end is observed to detach from the substrate, while the other two adsorbed groups remain bound to the surface throughout the simulation.

(38) Schiffrin, A.; Riemann, A.; Auwärter, W.; Pennec, Y.; WeberBargioni, A.; Cvetko, D.; Cossaro, A.; Morgante, A.; Barth, J. V. Proc. Natl. Acad. Sci. U.S.A. 2007, 104, 5279.

(39) Ireta, J.; Neugebauer, J.; Scheffler, M.; Rojo, A.; Galvan, M. $J$. Phys. Chem. B 2003, 107, 1432.

(40) Weigelt, S.; Busse, C.; Petersen, L.; Rauls, E.; Hammer, B.; Gothelf, K. V.; Besenbacher, F.; Linderoth, T. R. Nat. Mater. 2006, 5, 112.

(41) Pauling, L. Chem. Ind. (Suppl.) 1948, $1,1$.

(42) Koshland, D. E. Proc. Natl. Acad. Sci. U.S.A. 1958, 44, 98.

(43) All structural representations were obtained using the VMD software (Humphrey, W.; Dalke, A.; Schulten, K. J. Mol. Graphics 1996, 14, 33) 